\title{
Pratiques
}

Linguistique, littérature, didactique

191-192 | 2021

Théâtre contemporain : Écritures et Représentations

\section{Discours direct libre et parole intérieure}

Free direct speech and inner speech

\section{Alain Rabatel}

\section{(e) OpenEdition}

Journals

Édition électronique

URL : https://journals.openedition.org/pratiques/10832

DOI : 10.4000/pratiques.10832

ISSN : 2425-2042

\section{Éditeur}

Centre de recherche sur les médiations (CREM)

\section{Référence électronique}

Alain Rabatel, « Discours direct libre et parole intérieure », Pratiques [En ligne], 191-192 | 2021, mis en ligne le 15 décembre 2021, consulté le 03 janvier 2022. URL : http://journals.openedition.org/ pratiques/10832 ; DOI : https://doi.org/10.4000/pratiques.10832

Ce document a été généré automatiquement le 3 janvier 2022.

(c) Tous droits réservés 


\title{
Discours direct libre et parole intérieure
}

\author{
Free direct speech and inner speech
}

\author{
Alain Rabatel
}

1 Une double évolution caractérise les pratiques romanesques du $\mathrm{XX}^{\mathrm{e}}$ siècle, l'une vers la multiplication des marques, produisant des formes hybrides, l'autre vers leur raréfaction, voire leur disparition, l'absence de marquage typographique favorisant l'émergence des discours directs libres. Ces derniers, qu'on retrouve plus spécifiquement dans certains genres de discours écrits, littéraires et romanesques, posent maintes questions, en raison de leur nature :

2 Le discours direct est libre parce qu'il n'est pas introduit par un verbe, ni marqué typographiquement, mais il est contextuellement signalé. Les alternances de plans d'énonciation (temps, personne, deixis) inscrivent linguistiquement les discours directs libres dans le corps du texte. Parfois, cependant, son apparition demande davantage de travail interprétatif de la part du lecteur pour déterminer qui parle dans une séquence. [...] (Rosier, 1998, p. 295)

3 Je proposerai dans une première partie quelques éléments réponse dans le cadre théorique de référence pragma-énonciatif qui est le mien ${ }^{1}$. Dans une deuxième partie, je reviendrai sur la nature du discours direct libre, ses relations avec d'autres formes de discours « rapporté », notamment avec le discours direct, ses rapports avec le discours primaire quand ce dernier, en contexte narratif, est chargé de la diégèse. Enfin, dans une troisième partie, je réexaminerai son marquage et son fonctionnement textuel, dans le cadre d'une narration empathique qui épouse au plus près la subjectivité des personnages. Je serai ainsi amené à mettre en relief les relations que le discours direct libre entretient avec l'expression des pensées, voire de la parole intérieure (PI). En effet, ce discours apparait comme une de ses formes d'expression privilégiées ${ }^{2}$, soit dans de courts fragments en dehors de tout monologue intérieur (MI), soit dans des portions de texte relativement développées, au point de donner lieu à des embryons de MI, sans pour autant devenir des MI autonomes et sans occuper tout l'espace du texte. 


\section{Cadre théorique}

\subsection{Analyse pragma-énonciative}

4 Je m’appuie sur une approche énonciative héritée d'O. Ducrot (1984) ${ }^{3}$, distinguant le locuteur - en tant que source d'un acte d'énonciation réalisant une parole (ou du texte, à l'écrit) - de l'énonciateur, en tant qu'il est source, ou, à mieux dire, support des points de vue (Rabatel, 2012, 2018). La distinction est fondamentale pour l'étude des phénomènes dialogiques et polyphoniques dans lesquels opèrent diverses instances de locution, et, en outre, maints points de vue $(\mathrm{PDV})^{4}$ qui ne se laissent pas référer à une source locutrice quelconque et qui n'ont même pas l'apparence de discours ou de discours rapportés, à l'instar des points de vue perceptuels, des stéréotypes (Rabatel, 1998, 2008). Autrement dit, c'est à partir de l'étude de la référenciation des prédications, de celle des actes de langage explicites ou sous-jacents et des choix modaux (au sens de Gosselin, 2010) que l'on peut déterminer si la (ou les) prédication(s) exprime(nt) le PDV du locuteur/énonciateur premier (L1/E1) $)^{5}$ comme c'est le cas pour les énoncés narratifs pris en charge par le narrateur, ou renvoie(nt) au PDV (et donc aux modalités) du locuteur/énonciateur second ${ }^{6}$ (12/e2), à l'instar de la plupart des exemples cités ici

\subsection{Discours représentés, plutôt que rapportés}

5 Dans un de mes ouvrages (Rabatel, 2003, p. 73 ; repris dans Rabatel, 2008), je proposais d'« amender » la définition de L. Rosier (1998, p. 125) : le discours représenté (DR) sert à représenter le discours d'autrui, ce qui inclut les cas d'auto-citation, dès lors que le discours renvoie à une autre actualité du locuteur représentant $(=\mathrm{L} 1)$ soulignant la diversité des manifestations de cette représentation:

- selon la nature des phénomènes représentés, parole et/ou pensée et/ou perception ;

- selon la nature du lien entre le discours citant et le discours cité ${ }^{8}$;

- selon la nature de la représentation du « discours » cité : comme discours de $\mathbf{1 2}$ ou comme point de vue de e2, sans que ce dernier n'ait été représenté comme locuteur de son point de vue ;

- selon les formes de prise de distance envers telle ou telle manière de dire et/ou de penser et/ou de percevoir: distance temporelle, épistémique (perception, inférences ${ }^{9}$, dire d'autrui), axiologique, etc. (Rabatel, 2003, p. 73)

En effet, ce qu'on entend par « discours rapportés » est sujet à caution : la plupart de ces formes sont loin d'être des discours effectivement proférés par des locuteurs représentés. La co-présence, dans l'espace de l'énoncé (ou de plusieurs énoncés successifs) de deux espaces énonciatifs distincts n'implique ni antériorité ni réalité objective du discours représenté ${ }^{10}$ : il suffit que L1/E1 le convoque dans son propre discours pour que le DR ait une consistance linguistique. La problématique des " discours » représentés renvoie donc à la façon dont le locuteur représentant est en capacité de faire écho dans son propre discours aux PDV autres (ce qui inclut, vu la disjonction locuteur/énonciateur, des PDV indexés sur des paroles et d'autres, indexés sur des pensées autres ou sur des perceptions provoquant des inférences ${ }^{11}$ liées à des pensées autres), tout en les éclairant en fonction de ses propres buts communicationnels ou expressifs. J'emploie et répète intentionnellement le terme 
autre, pour faire entendre d'emblée que, pour moi, les PDV comprenant des pensées et des perceptions relèvent de plein droit de la problématique de l'altérité, a minima dans sa dimension auto-dialogique. En effet, leurs positions énonciatives ne coïncident jamais pleinement, mais dénotent des ajustements (à la réalité extralinguistique, à des dits ou à des dires antérieurs) relevant tantôt de la représentation des discours autres (RDA), tantôt de l'auto-représentation du dire (RDA) ${ }^{12}$. Pour insister sur l'importance stratégique de cette notion de représentation, je parlerai ci-après de discours représenté direct (DRD), de discours représenté direct libre (DRDL), de discours représenté indirect (DRI), de discours représenté indirect libre (DRIL), de discours représenté narrativisé (DRN).

\subsection{Présentation du corpus}

7 Le corpus est constitué de romans policiers de D. Manotti ${ }^{13}$, une auteure de romans noirs parmi les plus en vue en France. Il se compose des romans noirs suivants : Sombre sentier (1995, Paris: Le Éd. Le Seuil, désormais SS), À nos chevaux (1997, Paris : Éd. Rivages, désormais ANC), Nos fantastiques années fric (2001, Paris : Éd. Rivages/Payot, désormais NFAF), Racket (2018, Paris : Éd. Les arènes, désormais R), Marseille 73 (2020, Paris : Éd. Les arènes, désormais M73).

\section{Analyse de discours représentés directs libres (DRDL)}

Partant du constat que, «lorsqu'on se situe sur un plan textuel, l'absence de marques [du DRDL] est toute relative » (Rosier, 1998, p. 295), je voudrais revenir sur la nature du DRDL, compte tenu de la diversité de ses manifestations et de la variété de ses relations avec d'autres formes de discours représentés. Je m'appuie ici sur les ouvrages de L. Rosier (1998) et de J. Authier-Revuz (2020, XI, p. 143), à qui j'emprunte sa description formelle des modes du discours autre, fondé sur des traits différentiels : le DRDL et le DRD ont en commun de représenter l'objet du dire, de s'attacher à sa dimension signifiante (d'où sa nature d'autonyme) et de reposer sur deux ancrages énonciatifs différents, celui du locuteur représentant et celui du locuteur représenté. Les différences de formes d'expression entre DRD et DRDL apparaissent donc au niveau du discours ${ }^{14}$. Or, c'est un des enjeux de ce travail, les différences de fonctionnement textuel/discursif du DRDL par rapport à celui du DRD me semblent majeures, au plan interprétatif, une fois posée la parenté énonciative fondamentale au plan systémique entre DRD et DRDL. Le lecteur comprendra, au terme de ce texte, que mon accord avec J. Authier-Revuz est circonscrit à certaines formes de DRDL, qui ne sont pas les plus fréquentes dans mon corpus.

\subsection{DRDL dans une narration hétéro-diégétique au passé}

9 Comme L. Rosier l'a indiqué, le DRDL est une forme relativement récente, allant de pair avec l'abandon des récits au passé à la G. Flaubert ou à la É. Zola et avec l'émergence des récits au présent. Cela explique la relative rareté des DRDL dans les narrations hétérodiégétiques au passé; et, du même coup, la fréquence des DRDL chez D. Manotti, qui privilégie la narration au présent. Je cite malgré tout quatre exemples de DRDL en 
narration hétéro-diégétique au passé, dans des textes contemporains (qui ne sont pas de D. Manotti), avec « je » + présent en (1) et (2), « tu » + présent en (3), qui contrastent avec la narration au passé et en troisième personne :

(1) Le chauffeur vint une nouvelle fois informer Madame que la voiture de Madame attendait Madame et que, si Madame voulait bien se donner la peine, alors Madeleine fit un petit signe, merci Ernest, j'arrive, et dit, d'une voix qui exhalait le regret :

- Je vais devoir te quitter, Yvonne, je suis désolée... (Lemaître P. [2013]. Au revoir làhaut. Paris : A. Michel, p. 236) ${ }^{15}$

(2) M. Péricourt se levait fatigué, se couchait fatigué, je me traîne, pensait-il. (Lemaître P. [2013]. Au revoir là-haut. Paris : A. Michel, p. 374)

(3) Elle désigna le cordon, il approuva du regard, oui, d'accord, ne t'inquiète pas, elle vérifia le verre, la bouteille d'eau, le mouchoir, les pilules. (Lemaître P. [2013]. Au revoir là-haut. Paris : A. Michel, p. 185)

10 (1) et (3) sont des DRDL locutoires évoquant un acte de parole et l'oralisation de son contenu. On pourrait objecter (comme le fait un des relecteurs de ce texte) que les fragments en italiques sont plutôt la traduction verbale de signaux mimo-gestuels. C'est indéniable; mais il est tout aussi indéniable que cette traduction emprunte la forme de DRDL, avec ses caractéristiques déictiques. C'est cela qui (m')importe. Au demeurant, le DRDL, comme le DRD, n'implique pas toujours des paroles prononcées auparavant. Et rien n'interdit que le DRDL verbalise des gestes, voire les redouble. Assurément, les analyses linguistiques prennent peu en compte ces phénomènes, pourtant fréquents dans la réalité - et c'est un des intérêts du corpus que de présenter des formes inédites de DRDL. Certes, on pourrait rétorquer que, si les mimiques sont celles du personnage, leur verbalisation est due au narrateur : mais cela ne contredit en rien l'existence du DRDL, car la deixis est bien celle du locuteur second, non celle du locuteur représentant.

11 En revanche, (2) - qui comporte une incise, en position finale, après le DRDL - évoque un DRDL infra-verbalisé : il s'agit non d'un dire mais d'une pensée, vu le verbum putandi (Rabatel, 2003) qui prend la forme d'un DRDL. On pourrait appeler ce genre de DRDL indiquant un mouvement de pensée, une parole intérieure, un DRDL-PI. Ce genre de

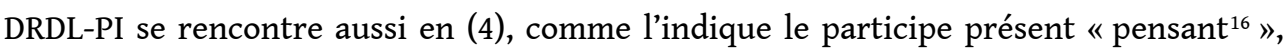
avant le DRDL :

(4) Le Coureur était en plein vol à présent, planant à cinquante-huit mètres par seconde - plus vite que la chute libre dans l'espace - se poussant jusqu'à la limite de la douleur, se torturant pour atteindre une frontière qu'il pensait ne jamais pouvoir franchir, repoussant encore la limite, aspiré en avant par la vitre de plexiglas, avalant l'air, craignant de s'enflammer à cause de la chaleur, craignant de se désintégrer sous la douleur, terrifié surtout par la peur, pensant, dieu en qui je ne crois pas aide-moi à continuer je t'en prie. Avançant plus vite que ses pensées, le Coureur s'abandonna à la vitesse, s'abîma dans la vitesse, devint la vitesse. (Littell $R$. [2012; 1975], Le cercle Octobre. Paris : Éd. Le Seuil, p. 23)

12 Là encore, le relecteur remarque que les exemples (2) et (4) sont complexes et problématiques - dont acte, c'est pour cela que je les ai choisis - et que l'incise de (2) et le verbe «pensant » en (4) orientent plutôt vers du DRD que vers du DRDL, en dépit de la ponctuation qui jouerait un rôle secondaire. Je crois discutable de considérer comme secondaire la ponctuation dans des textes qui jouent explicitement avec elle. Cela dit, l'objection est consistante, mais très problématique, parce que si l'on va au bout de ce raisonnement et que l'on tient pour secondaires les marques formelles, alors on ne peut que conclure que le DRD ne se limitant pas aux sempiternels deux-points-ouvrez- les- 
guillemets, il n'y aurait plus de différence entre le DRDL et le DRD. Or même si l'on peut lister un nombre très important de variations (Rosier, 1998, p. 208-219 en répertorie une petite trentaine de variantes), il n'en reste pas moins que a) : certaines sont plus prototypiques que d'autres; b) : le DRDL joue avec les conventions, et donc avec les formes prototypiques.

13 En somme, cette première brassée d'exemples met en relief les difficultés que pose l'existence du DRDL, si l'on adopte une conception large du DRD qui va jusqu'à englober les manifestations qu'on pense sous la notion de DRDL. Ces difficultés reposent aussi sur les liens complexes entre DRDL et narration ${ }^{17}$, pour les DRDL locutoires, ou encore sur les rapports que le DRDL entretient avec l'expression de la pensée, voire de la parole intérieure. Cette question est largement sous-estimée dans les travaux sur le DR en général et sur le DRDL en particulier. Il est ainsi significatif que l'ouvrage Aux marges des discours rapportés (Germoni \& Stolz, 2019), qui consacre pourtant une consistante première partie aux DRDL (également analysés en lien avec d'autres formes de DR dans les autres parties), s'attarde très peu sur la distinction DRDL locutoire et DRDL-PI, à l'exception notable d'A.-M. Paillet (2019, p. 48-49, 57).

\subsection{DRDL dans une narration hétéro-diégétique au présent ${ }^{18}$}

La narration au présent neutralise l'opposition entre le premier plan narratif correspondant à l'enchaînement des actions et le deuxième plan descriptif, commentatif ou explicatif (Combettes, 1992). Cependant, il n'y a rien de commun entre un présent de narration, qui peut permuter avec un passé simple, et une forme de second plan commentatif permutable avec un imparfait ou un autre temps de visée sécante (Rabatel, 1998, p. 30-50) ${ }^{19}$. Ces présents historiques sont donc très différents des présents de discours (f)actuels et ponctuels, dans le DRDL (en italique), par lesquels le personnage pense en son for intérieur à son patron, à la cadre américaine qu'il a rencontrée (passé proche) et à ce qu'il décide de faire (futur proche).

(5) Comme chaque matin en arrivant au bureau, Nicolas Barrot passe [passa] faire son rapport à Carvoux et prendre les consignes. Celui-ci l'accueille [accueillit] debout, le sourire aux lèvres.

- Alors, vous avez emballé la banquière?

Appliquez-vous, jeune homme, il se pourrait que le sort d'Orstam dépende de vos performances au lit...

Nicolas bafouille [bafouilla] :

- Autre chose, monsieur?

Non, Carvoux n'a [n'avait] rien d'autre à lui dire.

Nicolas s'enfuit [s'enfuit, au passé simple] dans son bureau, entre la honte et l'exaspération. Qui a pu lui dire ?...Il m'espionne ?... Ce ton... Je le hais. Il essaie [essaya] de travailler, attaque [attaqua] un rapport, n'arrive [arrivait] pas à se concentrer. S'il savait ce qu'elle dit de lui... incapable, nuisible, à éliminer. Parce que c'est lui, Barrot, qui a dîné avec July Taddei, pas Carvoux. C'est à lui, Barrot, que July a fait des confidences. La colère s'apaise [s'apaisa ou s'apaisait], peu à peu. Lui me méprise, pas elle : «faitesmoi confiance, Nicolas, je vous guiderai... » Au milieu des requins de la haute finance. Et j'emmerde Carvoux.

Il ferme [ferma] les yeux. Je ne serai pas frileux. D'abord, ne pas me faire laminer par ce type, qui me balancera à la première occasion. C'est décidé. J'enregistre tous nos entretiens. J'aurai le courage. L'arme fatale. Il n'y tient [tenait] plus. (Manotti D., R. p. 89-90) 


\subsection{DRDL, personnes allocutives et deixis}

Comme on le voit dans les exemples (6) à (12) ci-dessous, le DRDL est davantage marqué qu'en (5), dans la mesure où, si le contraste temporel entre formes de premier plan et de second plan est brouillée ${ }^{20}$ par la récurrence des présents - atténuant ainsi la distinction entre les plans de la narration (énonciation historique) et celui du discours (énonciation personnelle) ou des commentaires métadiscursifs -, la différence est néanmoins énonciativement marquée par le changement de personnes, avec narration en troisième personne et DRDL en première personne. Dans tous les cas, les changements de dénomination ou de pronom renvoient au même référent. Le contraste est à son maximum, dans les exemples (7) à (9), dans lesquels il y a changement, signalé par les gras, entre la dénomination relevant de la régie narrative et le « je » du DRDL, voire le «tu», si le «je» dialogue avec lui-même ${ }^{21}$, comme en (9). De plus, au plan énonciatif, la référence est déictique, renvoyant directement à la situation, comme il apparaît nettement avec le «ça » ((7)) ou « ici » ((10)), où avec la mention des noms et prénoms des personnes objets du discours, saillants dans la pensée de l'énonciateur, en (8) ou (9).

(6) Elle lui tend [tendit] la main pour sceller l'accord. Romero la prend [prit] et la porte [porta] à ses lèvres. Cette femme est dangereuse. Et je suis amoureux. (Manotti D., SS, p. 155)

(7) Soleiman ouvre [ouvrit] l'AG en demandant la confirmation, par les adhérents, de sa désignation comme secrétaire général (du comité négociant la régularisation des clandestins turcs du Sentier). [[Unanimité, par acclamations. Bouffée d'intense bonheur. $\left.{ }^{22}\right]$ ] Moi, dans ma vie, j'aurai connu ça, au moins une fois. (Manotti D., SS, p. 139)

(8) Sur le chemin du retour, Daquin rêvasse [rêvassa] un peu. Donc, Celik était un indic de Maillant. Je le dis, ou je ne le dis pas à Sol ? Et qui le savait? (Manotti D., SS, p. 171)

(9) Elle [Nadia] rejoint [rejoignit] sa chambre mansardée au sixième étage. [[Profond malaise.]] Cet Adel Khider ne réagit pas comme un homme en danger. Asensio et Picon me racontent des salades? Ils veulent savoir s'il est sur la piste du tueur de Malek, c'est tout. Peut-être quelqu'un qu'ils connaissent, qu'ils protègent? Ils te manipulent. Vite dit. Ils se servent de toi, d'accord. Mais ne sois pas naïve. Tu as une formation, un boulot, une chambre... Tout se paie dans ce monde. Tu préfererais qu'ils te baisent et t'exploitent, comme Asensio le fait avec ta mère? Ou tu préferes peut-être la misère? [[Impossible de dormir. Trop chaud sous les toits.]] (Manotti D., M73, p. 205)

(10) À chaque pas, Noria se sent [sentait] un peu lourde, la tête vide et sonore. Qu'est-ce que je fais ici?... Qu'est-ce que je suis venue chercher? Un écheveau de sentiments, de sensations, de regrets, de blessures, une tonne sur l'estomac. Pas envie de démêler, $\mathbf{j}$ 'y penserai demain. [[Trois étages, une marche après l'autre, en se cramponnant à la rampe.]] Quand elles arrivent [arrivèrent] sur le palier du troisième étage, Mohamed, le frère aîné de Noria, sort [sortit] de l'appartement de la mère en claquant la porte (Manotti D., R, p. 154).

(11) Elle [Noria] raccroche [raccrocha], livide, débranche [débrancha] le téléphone et vient [vint] s'asseoir sur le canapé. S'appliquer, elle est à moi. Noria enlève [enleva] son manteau, le dépose [déposa] sur le banc en bois. Puis elle s'installe [s'installa] dans un des fauteuils. [[L'autre ne parvient [parvenait] pas à protester.]]

- Qu'est-ce que vous me voulez? Vous n'avez pas fait le déplacement pour m'annoncer que j'étais plaquée? (Manotti D., NFAF, p. 179)

(12) Le Dem conduit [conduisait], calmement, nettement. Daquin, à ses côtés, rêvasse. Je n'aime pas la voiture. Une journée qui commence mal.

- Je suis allé rendre visite à Madame Moulin après que les gendarmes lui ont annoncé la mort de son mari. (Daquin subitement attentif.) Elle se retrouve avec un 
centre équestre sur les bras, alors qu'elle n'y a jamais travaillé. (Manotti D., ANC, p. 76)

Indépendamment du marquage énonciatif, le DRDL est éventuellement signalé par une syntaxe expressive - par exemple avec les mises en relief ou l'ordre des mots de (7), la répétition des questions ((8), (9), (10)), la présence des phrases simples (ou d'un flux verbal de phrases simples, comptant peu de mots), à quoi s'ajoutent un grand nombre de marques de subjectivité, notamment d'émotions ou d'affects - qui contraste avec une narration beaucoup moins saturée de subjectivèmes.

En raison du brouillage temporel, le DRDL est marqué par le contraste entre les personnes (grammaticales) délocutées de la narration hétéro-diégétique et la première personne du discours. À dire vrai, si le «je» domine massivement, la deuxième personne se rencontre aussi, même si c'est plus rarement. Plus rares encore, des formes de troisième personne, telles le « on », s'il a valeur de pronom personnel/indéfini inclusif, englobant aussi le «je », ainsi que l'ensemble des personnes perquisitionnant, en (13), ou prenant en compte les futurs manifestants qui respecteront les instructions données, en (14) :

(13) [[À gauche, dans des chemises cartonnées, des factures ou des tickets de Carte bleue : vêtements, bouffe, un bijou de chez Cartier à soixante mille francs. Peut-être la perle que portait Fatima Rashed quand elle a été assassinée ?]] On vérifiera. [[Des relevés de banque. Carnets de timbres, enveloppes, un tiroir plein de feutres, Bic, un stylo Mont-blanc, une bouteille d'encre. Un carnet d'adresses qui ne semble pas lui appartenir, et un trousseau de clés qui n'est pas celui de son appartement.]] On prend. [[Et un dossier salaires, avec le règlement des différentes piges qu'il donne aux journaux.]]

- Très à jour, remarque un flic.

Il en désigne une du doigt. (Manotti D., NFAF, p. 103)

(14) Finalement le consensus se fait. On peut cogner dur sur les melons si l'occasion se présente, mais, d'accord, pas de morts. La décision est donc prise de faire des petits groupes de vaillants citoyens pour secouer un peu tous les melons qui se risqueront à fréquenter la salle Saint-Joseph. Les gauchistes, on ne s'en occupe pas, les étudiants en médecine vont s'en charger. Ils se connaissent, ils ont l'habitude. Il est temps maintenant de passer au couscous. (Manotti D., M73, p. 160-161)

Beaucoup des exemples précédents montrent les liens très étroits entre DRDL et PDV perceptuels. Cette proximité confirme le rôle stratégique du PDV en tant que phénomène énonciatif (au sens où je définis ici l'énonciation comme source des PDV), et, subséquemment, accrédite la pertinence de l'hypothèse selon laquelle le PDV est une des formes de DR, hypothèse déjà étayée par la proximité entre PDV et DRIL (Rabatel, 2008). Bien évidemment, ces manifestations relèvent d'une représentation des discours autres qui ne concerne pas que des autres que soi, mais aussi des autres de soi, renvoyant à la mise en confrontation auto-dialogique d'un soi qui « dialogue » avec luimême, cherche à comprendre ce qu'il perçoit, s'interroge sur ce qu'il doit penser, dire, faire : d'où l'importance de la place des pensées dans le DR.

\subsection{DRDL sans personne explicite du dialogue}

Parallèlement aux DRDL signalés par des formes marquées contrastant avec le régime de la narration, il existe des DRDL moins signalés (et moins contrastés), davantage sujets à interrogation. On est alors fondé à se demander si on est face à un fragment de DRDL ou face à un commentaire du narrateur. Certes, il arrive que le contenu du DRDL 
oriente clairement vers une pensée représentée du personnage ${ }^{23}$, comme en (15) ou (16)

(15) Il descend [descendit] la rue du Faubourg-Saint-Denis jusqu'aux boulevards.

Passer [= il voulait passer, je veux passer] la nuit avec une fille, n'importe laquelle.

(Manotti, SS: 260)

(16) - Et le chauffeur au volant de la Mercedes, pendant que les deux autres

déménagent le cadavre, qui est-ce?

- J'ai aucune idée. Il est pas sur la photo.

- Aucune idée ? Vraiment? Ce ne serait pas Amadou Keny, par hasard?

[[Sakho sursaute, regarde Noria]], une sorcière cette femme. Il lâche :

- Oui, c'est peut-être bien lui, il m'a dit qu'il avait peur.

Sur l'ordinateur de Noria : «Bravo ». (Manotti, $R: 244$ )

La modalité désirante (" passer la nuit avec une fille») est celle du personnage, comme son indifférence à la personne ("n'importe laquelle »), non celle du narrateur.

En l'absence de marques de personnes et dans le doute que le contenu thématique du PDV soit nettement attribué au personnage, il est conjectural d'attribuer avec certitude le DRDL à une source locutrice et énonciative certaine. Ainsi, en (17), le fragment pourrait en première lecture être attribué autant à Delmas qu'à Daquin :

(17) P1 Il [Delmas] repasse à l'Évêché, discute avec Daquin. P2 La planque aura lieu

lundi. P3 Puis il [Delmas] va attendre sa compagne (...). (Manotti, M 1973 : 41)

Si c'est le commissaire Daquin qui informe Delmas, comme c'est le plus probable d'un point de vue hiérarchique, le fragment en italique correspond à un DRDL locutoire, transcrivant (sans les marques 'conventionnelles' du DRD ${ }^{24}$ ) ce que Daquin a effectivement prononcé. Mais rien n'interdit de penser que la narration focalise d'emblée sur l'intériorisation par Delmas de ce qu'aurait dit Daquin, dans un discours direct que la narration aurait en quelque sorte ellipsé, et qui serait récupérable par un calcul présuppositionnel du type: «Si X pense $\mathrm{Y}$, c'est que quelqu'un lui a dit $\mathrm{Y}$ auparavant, et si quelqu'un a pu l'informer, ce doit être son chef». Le tremblé interprétatif est réel, mais il n'affecte pas la nature du phénomène : c'est toujours un DRDL, mais dans le deuxième cas, ce serait un DRDL-PI, comme si Delmas se parlait à lui-même : « la planque aura donc lieu lundi », le « donc » faisant ainsi écho à un propos de Daquin au DRD que la narration ne rapporte pas. C'est l'hypothèse la plus vraisemblable, si l'on tient compte de la progression thématique des trois phrases successives : P1 et P3 sont en progression thématique à thème constant, avec le même personnage agissant en position thématique, et, dans ces conditions, il est plus économique, au plan interprétatif, de considérer que P2, enchâssé par P1 et P3, renvoie au même référent, donc corresponde au DRDL-PI de Delmas.

Faut-il en conclure que, puisqu'il y a absence de personnes allocutives, la deixis serait du même coup absente ? Dans l'exemple (16), « cette femme » a une valeur anaphorique par rapport à la réplique précédente de Noria, l'enquêtrice; je crois surtout que le démonstratif une valeur ana-déictique (Cornish, 2017), car la référence situationnelle est verbalisée, dans le contexte écrit. De même, en (15), « la nuit ", c'est la nuit à venir, saillante dans la psyché du personnage évoqué en troisième personne, et donc la référence est déictique, autant qu'anaphorique, la pensée d'une fille étant activée par les lieux de prostitution mentionnés juste auparavant avec les rues et boulevards. De même pour «la planque », saillante dans les pensées de Delmas, en (17). En d'autres termes, il existe des ana-déictiques, indépendamment de la présence des personnes allocutives, qui peuvent plaider selon le co(n)texte en faveur de l'hypothèse du DRDL. 
24 Mais les choses sont parfois plus complexes encore, le doute pouvant affecter la nature même du DRDL, et se croiser avec l'hypothèse que les fragments renvoient plutôt à des commentaires du narrateur. Personnellement, j'ai tendance à interpréter les fragments ci-dessous comme des DRDL, en vertu de la lecture empathique que je pratique ${ }^{25}$, et aussi en raison de mon intérêt théorique pour ces formes textuelles d'empathie linguistique - le chercheur ne doit jamais oublier les effets produits par ses goûts personnels comme par ses choix théoriques. Ainsi, en (18), il est difficile d'attribuer avec certitude le fragment en italique à une seule source déterminée : ce serait plutôt un DRDL-PI collectif, renvoyant au "soulagement» de l'ensemble des membres présents sur la tribune. La lecture empathique plaide en faveur de l'hypothèse DRDL-PI, rendant compte des états mentaux des actionnaires soulagés, après leurs craintes initiales, évoquées dans des DRN soulignés au préalable ${ }^{26}$.

(18) À la tribune, les administrateurs se penchent les uns vers les autres en murmurant, la main sur leur micro. L'un d'eux, Domenico Mori, un Italien, silhouette élégante et chevelure blanche romantique, prend la parole. Il dirige, en Italie, un consortium industriel métallurgique qu'il a construit lui-même à partir d'une affaire de famille. Son groupe est le plus gros actionnaire de la PAMA et le pivot sur lequel le Président a bâti son pouvoir. Et Mori est un ami personnel de longue date du Président, ils chassent ensemble le faisan en Tchécoslovaquie. Il est écouté dans un silence respectueux des milliards qu'il porte sur les épaules, avec un sentiment de soulagement à la tribune : tout va rentrer dans l'ordre.

- Nous n'avons aucune raison de nous opposer aux propositions défendues ici par monsieur Jubelin. (Léger accent italien.)

Frisson dans l'assemblée. Le Président, livide, crispé, murmure, en oubliant de cacher le micro : «Traître...comportement indigne d'un vieil ami... » (Manotti D., ANC, p. 9-10)

Le raisonnement peut être reproduit pour (19) : certes, les fragments renvoient au personnage centre de perspective, Bornand. En ce sens, c'est lui qui pense (PDRL-PI) qu' «il est temps de rejoindre Bestégui », que «Le président appréciera» le cadeau et aussi que la personne qui l'apporte soit celle qu'il avait remarquée (et appréciée) lors de sa promenade matutinale :

(19) Il est temps de rejoindre Bestégui au restaurant. Sur le chemin, un détour par la boutique du couturier devant laquelle le président s'est arrêté ce matin. Il [Bornand] achète une écharpe en vigogne, et charge la jolie vendeuse brune de la porter à l'Élysée dans l'après-midi. Le président appréciera. (Manotti D., NFAF, p. 32) Ailleurs, c'est l'organisation du texte qui entre en jeu. En (20), par exemple, l'appréciation sur le travail d'Attali correspond bien à un DRDL-PI. Mais la présence du narrateur est forte, à travers la suite de l'évocation des actions : les phrases nominales (gras) traduisent une narration nerveuse, disent la rapidité des actions, le caractère de script conventionnel de ce genre de situation où des policiers appartenant à des équipes différentes sont incités à travailler ensemble et échangent des propos convenus, tant ils ont de réserves à collaborer :

(20) Sur son bureau, Daquin trouve le paquet de photos apporté par Attali. Du bon travail.

Arrivée de Thomas et de Santoni. Présentations, poignées de mains. Des proches de Meillant, Daquin le sait. Thomas et Meillant se sont connus dans la Résistance et sont entrés ensemble dans la police, comme gardiens de la paix, en 1945. Mais Thomas n'a pas voulu, ou n'a pas pu, devenir commissaire. Il est, et restera, inspecteur divisionnaire, et il en ressent une amertume qui imprègne toute sa personne. (Manotti D., SS, p ; 33). 
«Présentations » est un DRN. Le narrateur est la source des premières informations sur Thomas et Santoni («Daquin le sait»); ensuite, il empathise sur Daquin dans les phrases suivantes, au DRDL-PI ${ }^{27}$. De ce fait, le lecteur infère que la coopération ne sera pas sans arrière-pensée, ce qui correspond au PDV de Daquin ; mais c'est aussi le PDV du narrateur, par-dessus celui du personnage, donnant à voir sa connaissance des arcanes de la police, des compétitions entre services, de leurs luttes d'intérêt, en fonction des proximités de leurs chefs avec tel ou tel pouvoir, politique, judiciaire, médiatique ou économique.

Au total, il apparait nettement que le DRDL est une forme complexe, dont le repérage est parfois plus ou moins difficile et dont l'attribution de la source énonciative l'est tout autant ${ }^{28}$. La difficulté, redoublée par l'absence de marques (2.3) ou par un surcroit de marques divergentes (2.4), est encore accrue lorsque le DRDL est souvent associé avec d'autres formes de discours représentés (2.5), dont il n'est pas toujours aisé de le distinguer. On comprend que, dans ces circonstances, le DRDL serve à exprimer autant des paroles que des pensées singulières ou collectives, voire doxales (Chaudier, 2019, p. 313).

\subsection{Le DRDL et ses relations avec les autres formes de DR}

29 Comme le montrent maints exemples ((1) à (4), (16), (19)), le DRDL contraste souvent avec des fragments narratifs. Il est aussi fréquemment associé à une ou plusieurs autres formes de discours représentés dans un co-texte étroit, à gauche ou à droite. Ainsi, en (21), le DRDL-locutoire, adressé par Daquin à Soleiman, son amant, vient après des fragments de discours narrativisé, soulignés.

(21) Daquin expédie les affaires courantes. Contacte ses collègues suisses et allemands. Accord. Courrier suit.

Un rapport sur Euroriencar, pour obtenir la mise en écoute téléphonique et la surveillance par les Stups.

Coup de fil à Soleiman. Ce soir à la maison. Plus de danger. Un flic devant la porte, je t'expliquerai. Débrouille-toi seulement pour qu'il ne voie pas ton visage. (Manotti D., SS, p. 158)

En (22), le DRDL est annoncé par un PDV perceptuel entre doubles crochets ouvrants et fermants, suivi par un DRD, dont le contenu est reformulé par une forme entre crochets simples, que l'on peut analyser comme un DRIL en troisième personne peu 'prototypique' ${ }^{\prime 29}$, vu l'absence d'incise et la présence des temps déictiques (présent et futur) :

(22) Daquin, en arrivant, regarde le dossier à son tour. [[Ça a l'air d'un travail rapide et bien fait. Signé Conrad.]] Ce connard essaie de se racheter.

- Vous avez l'air crevé, patron, asseyez-vous. Pour une fois c'est moi qui vais faire le café.

Daquin s'assied, se laisse aller. [C'est vrai qu'il est fatigué. Un coup d'œil à sa montre : dans trois ou quatre heures, il baisera Soleiman.] (Manotti D., SS, p. 161)

31 L'important n'est pas d'identifier des formes comme un entomologiste, il est de comprendre le glissando des perceptions, vers une pensée ou une verbalisation plus réflexive (voir infra, 2.6). Le DRDL a l'immense avantage de faire pénétrer au plus près dans les univers des personnages sans interrompre trop lourdement le récit, grâce à une narration empathique menée tambour battant. On retrouve une même proximité entre DRDL et DRIL en (23). 
(23) Avant de partir, Béchir salue Berger.

- Je te confie la famille Khider. Prends-en soin.

Puis Béchir disparaît. Berger râle. [Quelle condescendance... Ses grèves lui montent à la tête ? Non, lui, il n'est pas là pour prendre soin de la famille Khider, il est là pour la faire gagner, pour l'emmener jusqu'à l'arrestation des assassins, le procès, des condamnations, parce que seule la victoire est belle, seule la victoire est porteuse d'avenir, la défaite, le non-lieu, l'affaire classée sont interdits.] Il se sent vaciller. Ne pas fléchir. Je penserai à tout cela demain, à tête reposée. (Manotti D., M73, p. 172)

Annoncé par le discours narrativisé, "Berger râle ", le DRIL, entre crochets, est une réponse dialogique à une requête à laquelle l'avocat refuse de déférer. La réaction excédée prend d'abord la forme du DRIL en troisième personne, comme si Berger, l'avocat, voulait objectiver sa conception du rôle offensif de l'avocat, pour qui seule compte la victoire. Cependant la crainte d'une possible défaite s'insinue en lui, provoquant en réaction la décision («ne pas fléchir») qui s'extériorise ensuite en première personne, dans le DRDL-PI de la dernière phrase. En sorte que ces différents DR ont une dimension fortement dialogique les paroles étant des actes en réponse à d'autres paroles ou pensées qui interrogent le sujet dans son rapport à autrui, au monde comme à sa propre image.

En (24) - comme en (13), supra- le DRDL est associé à des fragments de DRIL entre crochets et de perceptions représentées (Rabatel, 1998), entre doubles crochets $^{30}$ :

(24) Daquin connaît bien le juge Bonnefoy, [il l'a pratiqué à plusieurs reprises depuis qu'il est en poste à Marseille, et il ne l'aime pas; il n'aime pas la morgue du personnage, la façon dont il affiche une honorabilité sûre d'elle-même pour masquer son être profond, sa lâcheté à toute épreuve.] Il me rappelle Vincent. Même étoffe d'homme. Pas fréquentable, Vincent. Peut-être, mais très baisable. [[Coup de chaud.]] Un peu de sérieux, commissaire.

Quand il se retrouve dans le bureau du juge, il est surpris. [[L'homme est atteint dans son physique, moins droit, moins rigide, un léger tic au coin de la bouche, les mains fébriles.]] (Manotti D., M73, p. 337)

Dès lors que le DRDL est associé à d'autres formes de DR, il est assez plausible de faire l'hypothèse, basée sur le mécanisme différentiel de la valeur saussurienne, que le DRDL prend aussi ses valeurs (DRDL-locutoire et DRDL-PI) du contraste avec son co(n)texte. Selon cette hypothèse, le DRDL-PI devrait contraster avec d'autres formes de DR, surtout des DR locutoires (DRD locutoires, DRI, DRIL locutoire, DRN) et le DRDLlocutoire contrasterait plus naturellement avec la narration. Mais ce ne sont là que des tendances, contredites par des contre-exemples. Ainsi, l'exemple (2) a beau côtoyer la narration, c'est un DRDL-PI, et non un DRDL-locutoire. Quant aux valeurs du DRDL lorsqu'il est associé à d'autres formes de DR, elles dépendent fortement de la prise en compte d'autres paramètres : a) : l'association du DRDL à un autre ou plusieurs autres DR ; b) : le fait que ces DR expriment des paroles ou des pensées c): la place du DRDL, avant tel autre DR ou après (voir infra (26)), ou encore son enchâssement dans tel DR ((21)). Il serait utile de procéder à des investigations systématiques de ces relations, si possible sur de grands corpus, de façon à avoir des données quantitatives significatives. (Encore qu'en ces matières, les valeurs dépendent de contrastes si fins et si complexes, en discours, que les approches qualitatives semblent davantage pertinentes.) 


\subsection{Bilan d'étape} manifestations discursives. Je ne veux pas m'attarder ici sur le fait que le DRDL est plus ou moins marqué par rapport au DRD, question importante, mais bien documentée : ainsi, le DRDL est le plus proche du DRD lorsqu'il repose sur la suppression des marques typographiques conventionnelles du DRD, deux points, tirets, guillemets. Le DRDL s'éloigne du DRL lorsqu'il repose sur l'absence de verbum dicendi, de personnes de l'interlocution et de verbes conjugués aux temps du discours, comme le signale L. Rosier dans la citation qui ouvre cet article. Ces points sont nettement soulignés chez K. Germoni et C. Stolz (2019), aussi chez S. Vaudrey-Luigi (2019), C. Stolz (2019, p. 136) et A. Laferrière et C. Narjoux (2019, p. 229-235). En revanche, je souhaite mettre l'accent sur d'autres éléments qui soulèvent des difficultés. Le DRDL est complexe, en effet, à plusieurs titres, en lui-même et dans ses relations avec d'autres formes de DR que le seul DRD.

1. Parce qu'il est libre, en un sens très différent du DRIL : la plupart des occurrences de DRDL apparaissent dans des phrases autonomes, complètes, pleinement actualisées dans une énonciation de discours. Seuls quelques exemples (e.g. (4), (16)) sont insérés dans des phrases relevant de la narration. Le DRDL se caractérise donc dans la plupart des cas par une triple autonomie, syntaxique, sémantique et énonciative, avec deux énonciations différentes - au sens que J. Authier-Revuz donne à cette notion, équivalant à deux actes locutoires différents et à deux deixis différentes et juxtaposées. Si le DRIL est également libre, au sens où il est non subordonné, il ne repose pas toujours sur deux ancrages énonciatifs partagés, contrairement à ce qu'affirme J. Authier-Revuz (2020). Il y a bien deux ancrages partagés pour les DRIL locutoires. Mais lorsque les DRIL renvoient à des pensées, il n'y a qu'un seul ancrage énonciatif, celui de L1/E1, associé à une déliaison entre les modalités de L1/E1 et celles de e2. Autrement dit encore, dans les DRIL-PI, celui qui parle, c'est le locuteur premier, faisant entendre le PDV (mais non véritablement la voix) d'un énonciateur second ${ }^{31}$.

2. Parce qu'il correspond à des différentiels énonciatifs, modaux et dialogiques diversifiés. Dans le premier cas de figure, hétéro-dialogique, le changement de voix correspond à un changement de la voix de L1/E1 - qui, en contexte narratif, joue le rôle de narrateur - vers la voix d'un locuteur second, comme en (16), (19) ${ }^{32}$. Le changement de locuteurs peut également aller d'un locuteur second à un autre, comme en (1), (5), etc. Dans le deuxième cas de figure, le différentiel correspond à un changement de perspective énonciative, avec des façons différentes d'envisager tel ou tel référent, émanant d'une même source énonciative, au sens où la source coréfère en dernier ressort à une même instance énonciativo-référentielle (i. e. au même sujet parlant), comme dans les exemples (4), (19) à (28), infra : en ce cas, l'usage du DRDL correspond à des cas d'auto-dialogisation, autrement dit de dialogisme intralocutif ${ }^{33}$ dans lesquels le locuteur dialogue avec lui-même, dans une autre actualité, un autre espace, un autre univers, par exemple un univers contrefactuel, hypothétique. En l'occurrence, l'autodialogisation repose sur le passage de la pré-réflexivité des perceptions ${ }^{34}$ à la réflexivité des pensées et paroles qui les analysent et déterminent leurs conséquences en termes d'action (Rabatel, 2008, p.417-420, 440-449, 464-469), en sorte que la relation de l'énonciateur à l'objet-de-discours a changé de nature, parce que l'énonciateur a 
changé de place, de temporalité, de cadres de référence (culturels, scientifiques, idéologiques, cultuels, esthétiques, éthiques, etc.).

3. Parce qu'il renvoie à deux valeurs sémantiques différentes : dans un cas, le DRDLlocutoire correspond à des paroles effectivement prononcées, comme dans les exemples (1), (3), (13), (14), (21) $)^{35}$. Dans l'autre cas, le DRDL-PI renvoie à des pensées : ainsi en est-il des exemples (2), (6) à (12), (15), (18) à (20), (22) à (28).

4. Parce que le fonctionnement textuel du DRDL donne lieu à au moins deux grandes articulations. Il est possiblement enchâssé et, en ce cas, il l'est tantôt dans la narration, e.g. (1) à (4), (16), (19), tantôt dans des répliques de DRD comme dans les deux exemples ci-dessous :

(25) - Tu témoigneras ? (Le Yougo a peur.) Écoute-moi bien. (Manotti D., SS, p. 37)

(26) - Nous savons qu'il était venu rencontrer son banquier. Il ne vous en a pas parlé?

- (Là, ça chauffe.) Pas du tout. Nous n'avons parlé que de souvenirs et fait des

blagues.

- Il vous a semblé dans quel état d'esprit?

- Détendu... normal. (C'est trop. En fuite, mafias... Ne pas surjouer.) (Manotti D., $R$, p. 275)

40 Il est plus souvent concaténé avec d'autres formes de discours représentés : e.g. en (1), avec un DRDL en position intermédiaire entre un DRI et un DRD; en (5), avec la concaténation de DRD et de DRDL ; en (16) avec une suite PDV + DRDL + DRD ; en (18), (20), (21), avec une suite DRN + DRDL ; en (22), avec un enchaînement PDV + DRDL + DRIL ; en (23), autour d'un enchaînement DRN + DRIL + DRDL, etc. Ces enchâssements ou concaténations de formes de DR alimentent la thèse d'un continuum (Rosier, 1998, 2008 ; Rabatel, 2008) et provoquent de savants effets de glissandos entre les diverses formes de DR et surtout entre ces formes et la narration proprement dite - a fortiori si elle est traversée par une écriture empathique grâce à laquelle le narrateur est au plus près de ses personnages, sans jamais abdiquer son propre point de vue.

Certaines des caractéristiques précédentes se cumulent : c'est le cas des DRDL-PI, qui correspondent dans la plupart des cas à un changement de perspective énonciative, par exemple au passage d'une perception (avec ses inférences) à une pensée ou à une parole plus réflexive ${ }^{36}$; c'est aussi le cas pour un DRDL éventuellement suivi d'une action (racontée par le narrateur, mais du point de vue de l'énonciateur personnage). Ces deux articulations ne sont pas sans effet sur la relation entre DRDL et PI, comme on va le voir à présent.

\section{Le DRDL, une forme d'expression libre propice à l'expression de la parole intérieure}

L. Rosier évoque un lien «historique » entre monologue intérieur (MI) et discours direct libre :

La tendance à l'actualisation décrite précédemment apparaît également comme historiquement marquée par l'usage d'un présent narratif dans les romans du $\mathrm{XX}^{\mathrm{e}}$ siècle: en effet, on peut affirmer que le discours direct libre est une forme linguistique dans les romans inaugurant un nouveau mode narratif par rapport à la narration du XIX ${ }^{\mathrm{e}}$ siècle à la Flaubert. [...] On quitte une narration dominée par le couple imparfait/passé simple et la troisième personne pour un discours direct libre combinant temps présent et personnes allocutives. L'évolution d'une prose au 
présent rend caduque l'usage spécifique du présent au profit de la personne.

(Rosier, 1998, p. 296) une signification de concomitance, alors il concerne plutôt les relations - dans le dernier quart du XIX ${ }^{\mathrm{e}}$ siècle -, entre MI, DRIL et PDV perceptuels, au sens où A. Rabatel $(1998,2008)$ les a analysées. Encore convient-il de noter que les DRIL et PDV existaient avant l'émergence du MI ; mais ils ont pris dans ce contexte nouveau une signification inédite, qui se déploie plutôt dans la première moitié du XX $\mathrm{X}^{\mathrm{e}}$ siècle. Quant au DRDL, il se manifeste plus tardivement, dans la deuxième moitié du $\mathrm{XX}^{\mathrm{e}}$. Qui plus est, ces liens concernent une notion, la parole intérieure (PI) ${ }^{37}$, plus qu'une forme, le MI. De plus, la PI ne se réduit pas à la " pensée discontinue ", elle peut tout à fait se développer dans des flux de conscience organisés, cohérents. La PI, en raison de la diversité de ses manifestations, se dit autant à travers le MI (autonome ou enchâssé) qu'à travers des formes d'expression plus extériorisées, voire spectacularisées, concernant un dialogue entre soi et soi (Rabatel \& Masi, 2020). Enfin, lorsque la PI prend le truchement des MI, ces derniers peuvent comprendre toutes les formes de DR, y compris des perceptions représentées (Rabatel, 1998, 2001).

La plupart des exemples précédents correspondant à des DRDL-PI (excepté (5) et (9)) sont courts : ils se composent au mieux de quelques phrases, généralement de phrases simples voire de phrases nominales courtes, de quelques mots (une huitaine au maximum, parfois beaucoup moins comme en (24)), l'ensemble n'excédant pas deux lignes. Ces DRDL-PI particulièrement peu développés sont particulièrement aptes à rendre compte de fulgurances de la pensée, de réactions immédiates à des paroles, le plus souvent au DRD ((5), (16)). Parfois le DRDL-PI suit un DRIL, comme en (23), (24) des perceptions (22)) ou des actions ((6), (20)). Ces réactions peuvent être quasiinstinctives, pré-réflexives, mais aussi tout à fait rationnelles et élaborées, si la pensée s'appuie sur des perceptions ou sur un savoir antérieur, comme en (20). Mais elles peuvent être aussi beaucoup plus développées, et c'est ce sur quoi je voudrais mettre l'accent. Autrement dit, les agencements dépendent des conditions dans lesquelles les enquêtes sont menées ${ }^{38}$. En l'occurrence, le DRDL-PI a à voir avec la délibération propre à l'enquête policière, aux pistes possibles qu'il faut vérifier. C'est le cas des extraits relativement longs et homogènes de $(27)^{39}$ et de (28) - dans ce dernier cas, le DRDL se déploie sur toute la fin de la page 165 et sur la suivante - :

(27) La nuit est tombée. Calme absolu dans le passage du Désir. Prendre le temps de réfléchir. Je suis encore en plein brouillard, mais, au moins, j'ai plusieurs pistes. Moreira et la mise en place de la filière? VL, Lestiboudois, le club Simon et la revente? Anna Beric et le ramassage du fric? Mais rien qui puisse me raccrocher à la mafia et à l'extrême droite turque. Sauf peut-être, une fois, la présence de la banque de Chypre et de l'Orient. Rester modeste dans ce rapport.

D'abord, les résultats concrets obtenus par Attali et Romero : quelques mots sur Moreira et Martens, pour appuyer une demande de mise sur écoute téléphonique, le plus tôt possible. Rien sur les méthodes employées, évidemment. Ensuite, Bernachon-Aratoff, c'est déjà fait. 
Tout sur la combine Simon. La liste des clients. Bien obligé. Les réactions en haut lieu ne vont pas tarder à se faire attendre. Les deux affaires doivent rester sous la responsabilité de mon groupe, la présence de Virginie Lamouroux, comme celle de Moreira, montrant qu'elles sont liées au trafic de drogue? Rien sur la CX et l'éventuelle filature. Attendons. Quant à Anna Beric et Meillant, ça, c'est mon jardin secret.

Terminé. Il est plus de 11 heures du soir. Fatigue. Déposer le rapport en remontant vers la maison. Et véritable regret de ne pas retrouver Soleiman. Souvenir du corps endormi sous la couette orange. La peau bronzée et le sexe plus foncé, presque noir. Pas la peine de rentrer dîner. Une choucroute dans une brasserie sur le chemin fera l'affaire. (Manotti D., SS, p. 115-116)

(28) Une fois le petit déjeuner avalé, Daquin s'allonge sur le canapé, les pieds en l'air, Europe 1 en bruit de fond. Deux heures devant lui. Réfléchir. Soleiman tourne un peu dans la maison avant de s'en aller, Daquin ne le voit plus et ne l'entend plus. Kashguri. Un entretien ${ }^{40}$. Pas encore un interrogatoire. Déjà un combat? Non, plutôt faire connaissance? Je sais trop peu de choses pour l'affronter.

J'ai cinq personnages. : Sobesky, VL, Kashguri, Anna Beric et Baker. Ils sont tous dans la course. Je ne sais pas dans quel ordre. Et je ne connais même pas les rapports qu'ils entretiennent entre eux. (Manotti D., SS, p. 165) celui de DRDL-pensée. Si penser, c'est dialoguer avec soi, c'est d'abord (d'un point de vue ontogénétique) dialoguer avec les autres; cette activité peut prendre des formes variées, les unes élaborées, spéculatives et déconnectées des contingences de la vie pratique et de l'action, les autres au contraire en prise directe avec le poids de la vie quotidienne : dans tous les cas, la pensée est dialogue avec autrui puis dialogue avec soi. Mais dans le contexte des romans noirs de D. Manotti, même si les enquêteurs travaillent en équipe, ils sont souvent seuls : seuls pour interpréter les indices; seuls lorsque l'enquête se heurte à des obstacles puissants -, pour savoir ce qu'ils peuvent dire à leurs collègues, aux autres services, à leur hiérarchie ; seuls pour déterminer les suites pratiques à donner à leurs investigations. Dans ces conditions, l'exercice de la pensée confine à la PI. Bien d'autres dénominations ont tenté de rendre compte du phénomène, mais la dimension intérieure de cette parole en soi est fondamentale (voir aussi Bergounioux, 2004 ; Santone, 2009) dans mon corpus, d'où le choix, ici, de DRDLPI.

Toutefois, la centration sur les liens entre le DRDL et la PI, particulièrement justifiés dans mon corpus - comme d'ailleurs le DRIL et les PDV perceptuels -, ne signifie pas que, de façon plus générale, il ne soit pas pertinent de souligner les rapports beaucoup plus vastes du DRDL avec la problématique théorique de la représentation des pensées, qui traverse toutes les formes de DR, et pas seulement le DRDL. Comme le montrent D. Vincent et S. Dubois (1997) : 63 et aussi S. Marnette (2005, p. 44-45), toutes les formes de DR sont traversées par la possibilité de reproduire fidèlement des paroles, ou d'être des pseudo-reproduction fidèles, de les inventer (sciemment ou non). Et il en va de même pour les pensées : le locuteur représentant peut les représenter fidèlement, il 
peut imputer à e2 des pensées fausses, de bonne ou de mauvaise foi. Rien ne serait plus faux que de cantonner des formes de DR à la représentation des paroles, d'autres à celle des pensées, puisque tant le DRD que le DRDL, tant le DRI que le DRIL ou le DRN peuvent évoquer des paroles ou des pensées. L'expression des "pensées » fait donc partie de plein droit de la problématique des discours représentés, au titre d'une parole infraverbalisée, subvocalisée, dont les échos provoquent de profondes résonnances en soi et en l'autre, d'une parole qui est à la source d'un espace mental autre que celui de la vie quotidienne, qui entre en dialogue avec lui, et qui est par nécessité représentée par L1/ E1, en contexte narratif hétéro-diégétique. C'est pourquoi je reviendrai plus centralement sur cette question à propos d'une future étude sur le DRN (Rabatel, en lecture, a et $b$ ).

Arrivé à ce terme, un certain nombre de questions interrogent le puissant modèle déployé par J. Authier-Revuz (2020), qui ne repose pas sur la disjonction locuteur/ énonciateur, privilégie la question de la deixis dans son abord de l'ancrage énonciatif, et traite assez peu de l'ancrage modal - évoqué pour certains exemples de DRIL (Authier-Revuz, 2020, p. 109-114 et 134) -, et, enfin, fait reposer l'immense majorité de ses exemples sur des paroles, et non sur des pensées. On imagine sans peine sa réponse: les pensées sont des énonciations, donc le modèle est préservé, et la nécessaire prise en compte des variations formelles (et de leurs effets) relève des actualisations en discours du modèle. Cependant, on entrevoit ici des points d'achoppement pour le modèle lui-même.

- Si le DRDL rapporte des pensées de e2, si ces pensées sont reconstruites empathiquement par L1, y a-t-il bien toujours selon la belle formule de J. Authier-Revuz (2020, p. 130) « deux chez soi énonciatifs $»^{41}$ ? On peut répondre par l'affirmative, mais à la condition de spécifier ce qu'on entend dans chaque cas par " espace énonciatif $»^{42}$ : dans le cas des DRDL locutoires, il y a deux espaces énonciatifs renvoyant à deux actes de locution (ou d'énonciation, dans les termes de J. Authier-Revuz); dans celui des DRDL-PI (ou pensée), il n'y a qu'un espace énonciatif locutoire et deux espaces 'énonciatifs' modaux différents, l'espace modal de l'énonciateur représenté dans l'univers de discours étant une construction (empathique) du seul locuteur représentant.

- Si l'on peut accepter la dimension autonymique des paroles représentées directement, DRD, peut-on encore défendre la dimension autonymique des pensées? Quelle est la pertinence de l'opposition usage/mention, dans ce cas ?

Ces questions portent sur les constituants abstraits du modèle. Si je maintiens mon accord avec le fait que le DRDL est une forme de DRD, je le circonscris aux DRDLlocutoires. Mais je considère que le DRDL-PI, comme d'ailleurs le fait que la plupart des formes de RDA peuvent exprimer aussi des pensées (DRD, DRI, DRN, DRIL, excepté les modalisations en assertion seconde et les modalisations autonymiques d'emprunt) invite à compléter le modèle magistral du discours autre, et, plus largement, du dialogisme. À suivre ${ }^{43}$, donc... 


\section{BIBLIOGRAPHIE}

\section{Sources primaires}

MANOTTI D. (1995). Sombre Sentier. Paris : Éd. Le Seuil.

MANOTTI D. (1999). À nos chevaux. Paris : Éd. Rivages.

MANOTTI D. (2001). Nos fantastiques années fric. Paris : Éd. Rivage/Payot.

MANOTTI D. (2018). Racket. Paris : Les Arènes.

MANOTTI D. (2020). Marseille 1973. Paris : Les Arènes.

\section{Sources secondaires}

AUTHIER-REVUZ J. (2020). La Représentation du discours autre. Berlin/Boston : De Gruyter.

BERGOUNIOUX G. (2004). Le Moyen de parler. Paris : Lagrasse/Verdier.

CHAUDIER S. (2019). " Discours rapportés et ethos narratorial dans deux romans contemporains ». in : Germoni K. \& Stolz C. (dirs). Aux marges des discours rapportés. Louvain-la-Neuve : AcadémiaL'Harmattan, p. 305-319.

CORNISH F. (2017). «SN démonstratifs et anadeixis : sens “spatial”, ou valeurs tributaires d'une stratégie pragmatique potentielle? ». Journal of French Language Studies, 27 (2), p. 215-239. En ligne : https://www.cambridge.org/core/journals/journal-of-french-language-studies.

DUCROT O. (1980). « Analyses pragmatiques ». Communications, 32, p. 11-60.

DUCROT O. (1984). Le dire et le dit. Paris : Éd. de Minuit.

GERMONI K. \& STOLZ C. (2019). Aux marges des discours rapportés. Louvain-la-Neuve : Éd. AcadémiaL'Harmattan.

GÉVAUDAN P. (2013). « Les rapports entre la modalité et la polyphonie linguistique ». in : Gévaudan P., Detges U. \& Atayan V. (dir.) Modalität und Polyphonie. Modalité et polyphonie. Modalidad y polifonía. Tübingen : Stauffenburg, p. 39-59.

GOLLUT J.-D. \& ZUFFEREY J. (2021). La Parole stylisée. Étude énonciative du discours indirect libre. Limoges : Lambert-Lucas.

GOSSELIN L. (2010). Les Modalités en français. La validation des représentations. Amsterdam/ New York : Éd. Rodopi.

JAUBERT A. (2019). « Main basse sur l'altérité : la problématisation énonciative des discours louches ». in : Germoni K. \& Stolz C. (dirs). Aux marges des discours rapportés. Louvain-la-Neuve : Académia-L'Harmattan, p. 295-304.

JORLAND G. (2004). « L'empathie, histoire d'un concept ». in : Berthoz A. \& Jorland G. (dir.)

L'Empathie. Paris : O. Jacob, p. 19-49. 
LAFERRIÈRE A. \& NARJOUX C. (2019). « D'un discours à l'autre dans Continuer de Mauvignier ». in : Germoni K. \& Stolz C. (dirs). Aux marges des discours rapportés. Louvain-la-Neuve : AcadémiaL'Harmattan, p. 227-244.

MAINGUENEAU D. (1986). Éléments de linguistique pour le texte littéraire. Paris : Bordas.

MARNETTE S. (2005). Speech and Thought Presentation in French: Concepts and strategies. Amsterdam : J. Benjamins.

PAILlET A.-M. (2019) «"Plonger dans la langue”. Le discours direct libre, zone de contact dans Corniche Kennedy de Maylis Kerangal ». in : Germoni K. \& Stolz C. (dirs). Aux marges des discours rapportés. Louvain-la-Neuve : Académia-L’Harmattan, p. 41-58.

RABATEl A. (1998). La Construction textuelle du point de vue. Lausanne/Paris : Delachaux et Niestlé.

RABATEL A. (2001). « Les représentations de la parole intérieure. Monologue intérieur, discours direct et indirect libres, point de vue ». Langue Française, 132, p. 72-95.

RABATEL A. (2003). «Les verbes de perception en contexte d'effacement énonciatif : du point de vue représenté aux discours représentés ». Travaux de linguistique, 46, p. 49-88.

RABATEL A. (2008). Homo narrans. Pour une analyse énonciative et interactionnelle du récit. Tome 1. Les points de vue et la logique de la narration. Tome 2. Dialogisme et polyphonie dans le récit. Limoges : Lambert-Lucas.

RABATEL A. (2009). « Prise en charge et imputation, ou la prise en charge à responsabilité limitée ». Langue française, 162, p. 71-87.

RABATEl A. (2012). «Positions, positionnements et postures de l'énonciateur ». Travaux neuchâtelois de linguistique, 56, p. 23-42.

RABATEL A. (2013). «Les relations Locuteur/énonciateur au prisme de la notion de voix ». in : Dufaye L. \& Gournay L. (éds). Benveniste après un demi-siècle. Regards sur l'énonciation aujourd'hui. Paris : Ophrys, p. 207-225.

RABATEL A. (2017). Pour une lecture linguistique et critique des médias. Éthique, empathie, point(s) de vue. Limoges : Lambert-Lucas.

Rabatel A. (2018) « L'énonciation culiolienne et la production du fictif ». in : Bedouret S. \& Copy C. (éds)., L'épilinguistique sous le voile littéraire. Culioli et la TO(P)E. Pau : Presses universitaires de Pau et des Pays de l'Adour, p. 115-136.

RABATEL A. (2019). « Empathie et récit ». Pratiques, 181-182. En ligne : http:// journals.openedition.org/pratiques/5655.

RABATEL A. (à paraître). «L'intrication des discours représentés et de la narration dans les romans noirs de Dominique Manotti ». Le Français Moderne, 90 (2).

RABATEl A. (en lecture a). « Retour sur la définition, les marques syntactico-énonciatives et modales du discours représenté narrativisé ».

RABATEL A. (en lecture b). « Des relations textuelles entre les discours représentés narrativisés et les autres formes de discours représentés et de la distinction entre discours représentés narrativisés exprimant des pensées et narration des états intérieurs ».

RABATEL A. \& MASI S. (2020). « La théâtralisation de la parole intérieure chez Camilleri, entre dialogue interne ou extériorisé du locuteur / écouteur plurilingue et humoriste ». Analele UniversităŢii din Craiova, Seria ştiinţe Filologice, XXIV (1), p. 193-235. En ligne : https://litere.ucv.ro/ 
litere/sites/default/files/litere/Cercetare/Activitate\%20stiintifica/

Analele\%20Facultatii\%20de\%20Litere/anale_2020_rezumate_fr.pdf.

ROSIER L. (1998). Le Ddiscours rapporté. Histoire, théories, pratiques. Bruxelles : Duculot.

VAUDREY-LUIGI S. (2019). « L'estompage entre discours direct et discours direct libre dans la prose de Maylis de Kerangal : une configuration idiolectale ?». in : Germoni K. \& Stolz C. (dirs). Aux marges des discours rapportés. Louvain-la-Neuve : Académia-L'Harmattan, p. 25-39.

SALVAN G. (2019). «Formes directes de discours rapporté et posture du narrateur dans La Disparition de Jim Sullivan de Tanguy Viel » in : Germoni K. \& Stolz C. (dirs). Aux marges des discours rapportés. Louvain-la-Neuve : Académia-L'Harmattan, p. 75-92.

SANTONE L. (2009). Egger, Dujardin, Joyce. Microscopia della voce nel monologo interiore. Rome : Bulzoni. SMADJA S. (2021a). La Parole intérieure. Qu'est-ce que se parler veut dire? Paris : Hermann.

SMADJA S. (2021b). Pour une grammaire endophasique, Vol. 1 Voix intérieures : un moi locuteur-auditeur. Paris : Hermann.

SMADJA S. (2021c). Pour une grammaire endophasique, Vol. 2 Une syntaxe, une sémantique et une prosodie de la conscience. Paris : Hermann.

SToLz C. (2019). «La portée figurale des formes de DD et DDL dans le roman du XXIe siècle », in : Germoni K. \& Stolz C. (dirs). Aux marges des discours rapportés. Louvain-la-Neuve : AcadémiaL'Harmattan, p. 127-139.

TISSERON S. (2017). Empathie et manipulations. Les pièges de la compassion. Paris : A. Michel.

VINCENT D. \& DUBoIS S. (1997). Le Discours rapporté au quotidien. Québec : Nuit blanche éd.

\section{NOTES}

1. Je remercie les relecteurs, dont les remarques m'ont permis de mieux faire entendre mes hypothèses. Bien évidemment, les éventuels points discutables restent miens.

2. Parallèlement à certains discours indirects libres exprimant des pensées, à certaines manifestations de points de vue perceptuels (Rabatel, 2001).

3. Je me sépare toutefois d'O. Ducrot sur l'instance de prise en charge qui est, d'après moi, l'énonciateur : voir la note 6.

4. L'expression PDV, sans la spécification perceptuel, est employée au sens le plus large du terme et indique le PDV de l'énonciateur sur l'objet-de-discours, quel que soit le sémantisme de l'énoncé, en sus de sa dénotation. Cette définition est beaucoup plus large que les PDV basés sur des perceptions, puisqu'elle concerne toutes les prédications (Rabatel, 2008, p. 79).

5. Les majuscules et le chiffre 1 codent le locuteur/énonciateur premier, les minuscules et le chiffre 2 les locuteurs énonciateurs/seconds, et le slash indiquent le syncrétisme entre $\mathrm{L}$ et $\mathrm{E}$ ou $\mathrm{l}$ et $\mathrm{e}$, c'est-à-dire les cas où les locuteurs expriment leur propre PDV. Contrairement à une tradition qui code avec le chiffre 1 le locuteur originel du DR, je confère le rôle premier au locuteur représentant qui cite les dires de 12 en jouant sur leur contextualisation et donc en pesant sur leur signification.

6. Sans compter les cas où le discours représenté renvoie au seul énonciateur représenté, dans le cadre des PDV sans parole : c'est le cas des PDV perceptuels, des 
discours indirects libres non locutoires, des discours directs libres non locutoires, ou encore des discours narrativisés non locutoires, qui expriment des pensées reconstruites par L1/E1, et non les paroles de 12. Ce qui revient à dire que c'est l'énonciateur (et non le locuteur) qui prend en charge les PDV, avec les modalités qui les accompagnent. Voir sur ce point Rabatel, 2009, 2012; Gosselin, 2010 ; Gévaudan, 2013.

7. Il est fréquent que les PDV des 12/e2 se superposent aux PDV de L1/E1: c'est particulièrement net avec les discours indirects libres, mais pas seulement (Rabatel, 2003, 2008).

8. J'utilisais alors la terminologie de D. Maingueneau (1986); avec le recul, il me semble plus juste et plus cohérent avec le paradigme des DR de parler de locuteurs représentant et représenté, d'autant plus que le terme de citation est polysémique et ne rend pas compte de la diversité des modes de représentation du discours autre (Authier-Revuz, 2020, p. 360-371). Le locuteur représentant correspond au locuteur premier, le locuteur représenté au locuteur second.

9. Un des relecteurs me signale que dans cette évocation des sources évidentielles, les inférences sont distinctes des perceptions, alors que dans mon ouvrage (Rabatel, 1998), j'insistais sur le lien entre perceptions et inférences. C'est un fait. Je ne me souviens pas des raisons qui m'ont poussé à écrire les choses de cette façon dans la citation cidessus. Avec le recul, je trouve que cette imprécision est très bienvenue, compte tenu de l'élargissement que j'ai apporté à la notion de PDV, dès le début des années 2000, en l'étendant à tout type d'énoncé, quel qu'en soit le contenu sémantique, et donc en ne la restreignant pas aux seuls PDV perceptuels (voir supra la note 6). Partant de là, on comprend qu'il soit utile de tenir compte des inférences tirées des perceptions et de celles qui sont indépendantes de ces dernières.

10. Voir les discours représentés directs (DRD) imaginaires, futurs ou hypothétique du genre "tu me diras: "XXX" ", "XXX", comme dirait Tartempion": même si ces discours n'ont pas été tenus au préalable, ce sont des discours que L1/E1 présente comme émanant d'un locuteur second.

11. Comme dans l'incipit célèbre d'Une vie, "Jeanne, ayant terminé ses malles, s'approcha de la fenêtre, mais la pluie ne cessait pas ", où le lecteur infère de la perception de Jeanne que la pluie contrecarre son désir de partir en voyage (Ducrot, 1980, p. 20).

12. Voir Authier-Revuz (2020) pour ces deux notions et infra, la fin de la section 2.3, les notes 32 et 33 ainsi que la conclusion.

13. Excepté les exemples de la section 2.1, qui illustrent d'un point de vue théorique un cas de figure n'existant pas dans le corpus.

14. Toutes les formes de représentation du discours autre (RDA) peuvent être à marqueur zéro (Authier-Revuz, 2020, p. 427).

15. Dans tous les exemples, les annotations sont de mon fait : le DRDL est en italique, le DRIL entre crochets ([xxx]), les perceptions représentées (des personnages) entre doubles crochets ouvrants et fermants ([[xxx]]) - signalant leur parenté avec le DRIL -, le DRN est souligné, les fragments en gras renvoyant à des commentaires grammaticaux particuliers.

16. Ce serait la même chose avec « se dire ». 
17. Accessoirement (par rapport à l'objet de ce texte), l'intrication des DRDL dans narration, ou, à mieux dire, la façon dont la narration épouse les motivations des personnages avant même de se déployer en DRDL, est une des caractéristiques du style de D. Manotti. On retrouve le même phénomène dans l'usage du DRN (Rabatel en lecture $a$ et $b$ ). Je traite plus complètement de ces stylèmes dans un ouvrage à paraître.

18. Un des relecteurs regrette que je n'aborde pas les DRDL dans une narration homodiégétique. Je comprends la frustration, mais outre que mon corpus ne comprend que des narrations hétéro-diégétiques, la question, passionnante, excède le cadre de cet article, déjà bien long. J'ajoute qu'il est de bonne politique de commencer par le type de narration qui objective le plus nettement les univers énonciatifs de L1/E1 et de 12/e2. Cela dit, la distinction locuteur/énonciateur est fondamentale pour rendre compte de la variation des positions énonciatives du je narrant et du je narré, alors que le locuteur renvoie toujours au même sujet parlant.

19. Les mêmes permutations seront effectuées en tant que de besoin dans les exemples suivants, dans le but de désambiguïser les effets du brouillage temporel.

20. "Brouillé ", mais pas neutralisé totalement, dans la mesure où il est toujours possible de récupérer, par des manipulations ou par l'analyse, les différentes valeurs fonctionnelles du présent.

21. Ce dialogue intériorisé peut même prendre la forme d'un embryon de délibération entre une troisième personne («s'appliquer ») et la première («moi»), en (11). Ce décalage entre la troisième personne et la première est étonnant; il produit un effet de distance avec la troisième personne et de proximité avec la première. J'explique cet effet par l'hypothèse que Noria s'adresse à elle-même en troisième personne sous la forme d'une injonction professionnelle (s'appliquer " = il faut s'appliquer dans ce genre de situation), la distance s'appuyant sur le déontique de la loi, avant de passer au « je » pour son intériorisation. Cette troisième personne n'est pas totalement délocutée, car elle pourrait permuter avec un «tu», mais au prix de l'adjonction d'une marque modale ( tu dois t'appliquer »).

22. Je ne développerai pas longuement les justifications pour lesquelles les fragments entre doubles crochets ouvrants et fermants correspondent à des PDV perceptuels, car cela excède mon propos. Dans les exemples (7) à (11), les PDV correspondent à des perceptions parfois de nature somesthésique, proprioceptive, dans des phrases nominales, le plus souvent, qui indiquent des réactions pré-reflexives, fulgurantes, des ressentis qui n'ont pas besoin, du point de vue de celui qui les ressent, d'être analysées dans des phrases complexes. Ces PDV sont pris en charge par l'énonciateur second, et non par le narrateur. De même en (28). Les notions de pré-réflexivité et de réflexivité sont présentées dans la note 34 .

23. Plus loin dans le texte, je parlerai de DRDL-PI, représentant la parole intérieure. J'insiste sur le fait que la PI est une notion psychologique et linguistique qui peut être exprimée à travers de multiples formes de DR, DRIL, DRDL, MI, PDV perceptuels associés à des inférences, discours narrativisés non locutoires (Rabatel, à paraître, Rabatel, en lecture $a$ et $b$ ).

24. Ces marques fussent-elles beaucoup plus variées que les simples /verbum dicendi $+\underline{:}^{+}$ «[...] $]_{\Perp} /$ qu'on retrouve dans toutes les grammaires. Voir Rosier, 1998, p. 208-219.

25. L'empathie correspond, en psychologie (Jorland, 2004), à un décentrement projectif par lequel un sujet se met à la place d'un autre, et essaie d'adopter ses manières de 
penser, en bonne ou en mauvaise part (Tisseron, 2017). Ces décentrages s'expriment en linguistique lorsque L1/E1 se met à la place d'un locuteur ou énonciateur second, imagine ce qu'il perçoit, pense, ressent, dire, fait dans les situations où il se trouve. Voir Rabatel, 2017, chapitre 2 et Rabatel, 2019.

26. Pour une analyse détaillée de ces DRN, qui ne sont pas des commentaires du narrateur, voir Rabatel, en lecture b.

27. Certes, l'hypothèse serait plus forte si le texte comportait deux points plutôt qu'un point après « Daquin le sait. "

28. C'est encore plus vrai lorsque les DRDL font écho à des PDV collectifs, anonymes, à des on-dit, des rumeurs (Rosier, 1998, p. 296). Mais mon corpus privilégie des usages plus individuels du DRDL, en lien avec le travail des enquêteurs, leurs hypothèses, leurs calculs, autrement dit avec la pensée ou la parole intérieure (voir infra, la partie 3).

29. C'est une façon de parler que je n'emploie que par commodité: ce sont les grammaires scolaires (et certaines grammaires universitaires) qui prônent cette thèse, démentie par l'immense variété des formes, selon les contextes, les genres, selon les discours oraux (Vincent \& Dubois, 1997), la prise en compte des dimensions prosodiques et mimo-gestuelles (Authier-Revuz, 2020, p. 335-342).

30. Sans compter, de surcroit, une superposition des PDV de L1/E1 et de 12/e2.

31. On retrouvera ce problème avec le DRDL-PI, en conclusion.

32. Cela correspond au RDA de J. Authier-Revuz 2020.

33. Dans l'auto-présentation du dire autre (ARD), les paramètres de l'énonciation (A : Acte d'énonciation; E : énoncé, $\mathrm{L}$ : locuteur; $\mathrm{T}$ : temps; Loc: localisation; CONT : contexte) coïncident avec ceux du discours autre (les mêmes paramètres écrits avec des minuscules), en sorte que dans l'ARD, A = a (et ainsi de suite), tandis que dans le RDA, A $\neq$ a (et ainsi de suite) (Authier-Revuz, 2020, p. 18-20 et 66). En d'autres termes, l'autodialogisation que j'évoque ici relève du RDA, vu le contexte. Mais il est bien sûr possible que l'auto-dialogisation corresponde à de l'ARD, lorsqu'un locuteur revient sur ses dires juste après les avoir formulés.

34. La pré-réflexivité est minimale lorsque l'objet perçu est d'emblée reconnu ; elle est de plus en plus réflexive lorsque ce dernier - rencontré pour la première fois, ou lorsqu'il est intrinsèquement complexe ou fait fond sur un environnement complexe (par exemple la perception d'un être animé qui émet des signaux contradictoires, voire d'un évènement par rapport à celle d'un objet inanimé) - nécessite des ajustements. Plus les ajustements comportent des mises en relation, des arguments, des marques expressives en lien avec la dimension cognitive, des dimensions méta-énonciatives ou méta-discursives, plus ils s'extériorisent dans une activité de parole, plus les PDV sont réflexifs (Rabatel, 2008, p. 417-420, 440-449, 464-469).

35. Dans ce cas, comme l'insertion du DRDL locutoire opère en s'insérant sans rupture apparente avec la narration, il est légitime de s'interroger sur les relations, en termes de postures énonciatives, entre L1/E1 et 12/e2 (Rabatel, 2012). Je n'ai pas la place de développer cette question passionnante, je renvoie à l'hypothèse la plus plausible, celle de la co-énonciation, analysée par G.Salvan (2019, p. 87-90). Mais on peut faire confiance au génie des auteurs (et, plus largement, à l'inventivité de tous les locuteurs) pour que d'autres cas se présentent, en sous- comme en sur-énonciation! 
36. Toute perception entraine toujours en contexte des inférences, rapportées au sujet modal (ou aux différents sujets modaux), qui s'inscrivent sur un gradient (voir la note précédente) et entrainent des interprétations.

37. Sur cette notion, voir Rabatel \& Masi (2020). Comme j'ai écrit le présent texte avant la parution des ouvrages de S. Smadja (2021, a, b et c) sur la parole intérieure, je ne les évoquerai pas ici, mais reviendrai sur eux dans une publication ultérieure.

38. Ils dépendent aussi des choix de l'écrivain qui organise sa narration en fonction de ses choix esthétiques, politiques, comme je le montrerai dans une publication complémentaire (Rabatel, à paraître).

39. Même s'il existe une différence entre le DRDL dans les deux premiers paragraphes en première personne et l'absence de première personne dans le dernier paragraphe, ce dernier correspond bien à un DRDL-PI de Daquin : outre la continuité sémantique du fragment, l'emploi du déictique « la (maison) » montre bien que le récit raconte à partir de la perspective de Daquin, la maison étant saillante pour le commissaire. Quant à la syntaxe, avec ses phrases nominales ou ses phrases simples courtes, elle est emblématique de la PI dont elle est censée rapporter la fulgurance des pensées (Rabatel, 2001 ; Rabatel \& Masi, 2020).

40. Pour l'analyse détaillée de ce DRN, voir Rabatel, en lecture b.

41. On peut répondre par l'affirmative, mais les espaces énonciatifs n'ont plus le même contenu.

42. Toutes choses égales, on en dirait autant à propos du livre de J.-D. Gollut \& J. Zufferey (2021), paru après l'écriture et l'évaluation de cet article, et sur lequel je reviendrai en détail dans un travail ultérieur. Car leur emploi de la notion d'énonciation, n'est guère précis en dépit de leur référence à 0 . Ducrot. De même pour la notion de voix, qui admet plusieurs définitions à la source de bien des flottements (Jaubert, 2019, p. 296-297; Rabatel, 2013). Il s'ensuit que leur conception de la bivocalité ou de la mono-vocalité est discutable: ce n'est pas la même chose que de mettre sous la notion de voix celle du locuteur exprimant son propre PDV d'une part, et, d'autre part, l'aptitude du locuteur à envisager des espaces mentaux différents, renvoyant à des énonciateurs/sujets de conscience différents, qui ne sont pas forcément locuteurs. Moyennant quoi, leur analyse du DIL comme forme mono-vocale renvoyant à la voix entendue dans le DRIL est partiellement erronée : lorsque le DRIL exprime des pensées, elles correspondent au PDV de e2, mais ce PDV est reconstruit par la voix de L1, qui est responsable de toutes les transpositions caractéristiques du DRIL : le DRIL-PI est donc mono-vocal, mais dialogique, en ce qu'il exprime le PDV de e2 (et le plus souvent le PDV de E1 sur le PDV de e2). En revanche, si le DRIL est locutoire, la thèse de la mono-vocalité ne tient pas.

43. Un des relecteurs pense que mes analyses ne remettent pas en cause la structure énonciative de la représentation des dires autres de J. Authier-Revuz, car elles « [plient] la réalité formelle de la manifestation linguistique (présence d'un je dans la plupart des énoncés examinés) au gré d'un présupposé selon lequel "l'espace mental du personnage [...] est par nécessité représenté par L1/E1, en contexte narratif hétéro-diégétique" ". Le débat est capital et l'objection robuste. Je crois cependant que ces je sont des projections; ces dernières ne concernent pas seulement les DRDL-PI, mais toute position énonciative. Ce débat ne peut trouver de réponse satisfaisante sur la base de cette seule étude. La réflexion est "à suivre", et ce n'est pas une affirmation 
rhétorique. Je poursuis la réflexion sur l'analyse du DRN (Rabatel, en lecture a et b). J'espère que le lecteur voudra bien patienter jusqu'à leur publication.

\section{RÉSUMÉS}

Résumé : Cet article revient sur l'analyse du discours direct libre, dans une optique pragmaénonciative. Il traite de la nature du discours direct libre, de ses marques énonciatives et textuelles, de ses relations avec les autres formes de discours représenté (en premier lieu le discours direct) et avec le discours primaire de la narration. Il distingue discours directs libres locutoires et discours directs libres exprimant des pensées voire la parole intérieure. L'article conclut sur un certain nombre de questions adressées au modèle de J. Authier-Revuz (2020) à partir de la prise en compte de la disjonction locuteur/énonciateur, de la multiplicité des supports de modalité et de la sous-évaluation des pensées dans les études du discours « rapporté ».

Abstract: This article revisits the analysis of free direct speech, from a pragmatic and enunciative perspective. It deals with the nature of free direct speech, its enunciative and textual marks, its relations with the other forms of represented speech (first and foremost direct speech) and with the primary speech of the narrative. It distinguishes between locutionary free direct speech and free direct speech expressing thoughts or inner speech. The article concludes with a number of questions addressed to J. Authier-Revuz's (2020) model, starting from the consideration of the speaker/enunciator disjunction, the multiplicity of modality supports and the undervaluation of thoughts in the studies of "reported" speech.

\section{INDEX}

Mots-clés : Disjonction locuteur/énonciateur, multiplicité des supports modaux, discours rapporté, discours représenté, espace mentaux, discours direct libre locutoire, discours direct libre-pensée.

Keywords : Enunciator/speaker disjunction, multiplicity of modal supports, reported speech, represented speech, mental space, locutionary free direct speech, thought-free direct speech.

\section{AUTEUR}

\section{ALAIN RABATEL}

Université Claude-Bernard-Lyon 1, ICAR, UMR CNRS 5191, Université Lumière-Lyon 2, ENS-Lyon 